\title{
Live Electrooptic Imaging Camera for Real-Time Visual Accesses to Electric Waves in GHz Range
}

\author{
Masahiro Tsuchiya ${ }^{1} \cdot$ Takahiro Shiozawa ${ }^{2}$
}

\begin{abstract}
Recent progresses in the live electrooptic imaging (LEI) technique are reviewed with emphasis on its functionality of real-time visual accesses to traveling electric waves in the $\mathrm{GHz}$ range. Together with the principles, configurations, and procedures for the visual observation experiments by an LEI camera system, the following results are described as examples indicating the wide application ranges of the technique; Ku-band waves on arrayed planar antennas, waves on a $\mathrm{Gb} / \mathrm{s}$-class digital circuit, W-band waves traveling both in slab-waveguide modes and aerially, backward-traveling wave along composite right/left-handed transmission line, and, waves in monolithic microwave integrated circuit module case.
\end{abstract}

Key words: Electrooptic Imaging, Electrical Wave Propagations, GHz Range, Real-Time Visual Access, Ultra-Parallel Measurement.

\section{Introduction}

Generally, it is highly beneficial to have prompt visual accesses to a phenomenon that is difficult to comprehend, which has been represented by the famous saying "seeing is believing". This is true also for electric waves at radio frequencies (RF) propagating in electrical/electronic circuitry, transmission lines, and radio wave media, particularly when they are in highly complex systems. RF electric waves are, however, inherently invisible in the space domain. Therefore, the relevant technological areas have been apart from the benefits provided by "experimental imagery for traveling electric waves".

This drawback has been tried to compensate so far by means of theoretical considerations and/or numerical simulations. Industrially important have been their usages to clarify origins of electrical malfunctions. However, a considerable amount of time and cost is known to be needed to arrive at appropriate models for satisfactory reliability in results of those theoretical methods.

Alternately, a spatial scan of an electric or magnetic field probe provides an image of an RF wave experimentally [1], [2]. Indeed, this method originates from the famous Hertz's experiments to first confirm electromagnetic waves. In spite of their recent progresses in sensitivity, invasiveness, spatial resolution, phase resolution, and frequency range, it takes generally a long time to construct an image, due to the unavoidable two-dimen- sional (2D) mechanical scanning. Therefore, prompt visual access is a benefit that is still far from achievable via this kind of imaging methods.

The scheme called as live electrooptic imaging (LEI) surpasses those probes in regards to the promptness of visual accesses [3], [4], which is realized by the high-degree parallelism for high frequency electrical measurements (Fig. 1). The LEI camera prototypes include 10,000 channels for their RF field measurements and real-time image displays. Extension of the parallelism up to 65,536 has been demonstrated recently [5].

On the bases of this LEI camera approach, it is now possible to "see" RF waves in real time and the benefits provided by the functionality of prompt visual accesses are ready for applications. In this paper, we review the recent progresses of the scheme demonstrated in a series of the LEI camera observations for traveling electric waves in the $\mathrm{GHz}$ range. The contents could imply future potentials of the LEI scheme for the high-frequency electromagnetic compatibility (EMC) issues. Here, subjects of the observations range from sub-GHz to $100 \mathrm{GHz}$ in frequency, from digital to analog in circuitry, and from guided to aerial in wave propagation modes. The configurations and operation procedures of the latest LEI camera prototype are explained as well as the principle of real-time traveling wave observation functionality.

\section{LEI Camera System}

\footnotetext{
Manuscript received May 16, 2011 ; revised November 10, 2011. (ID No. 20110516-04J)

${ }^{1}$ National Institute of Information and Communications Technology, Tokyo, Japan.

${ }^{2}$ Dept. of Communication Network Engineering, Kagawa National College of Technology, Kagawa, Japan.

Corresponding Author : Masahiro Tsuchiya (e-mail : mtsu@nict.go.jp)
} 
(a)

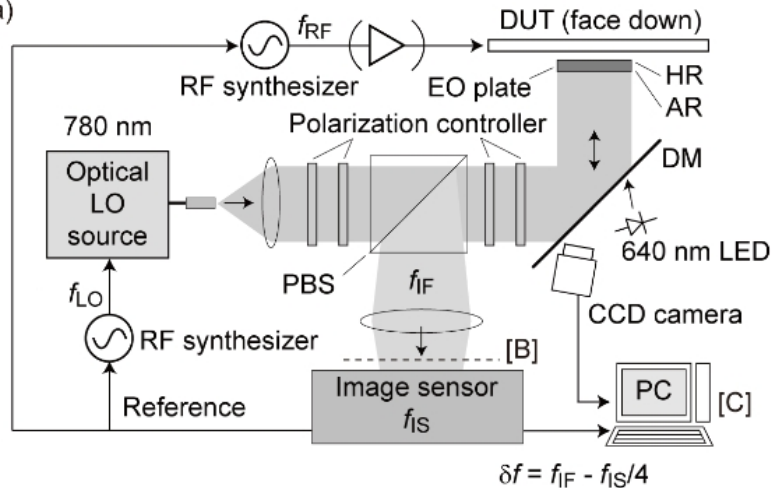

(b)

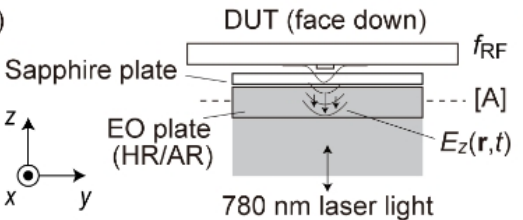

(c)
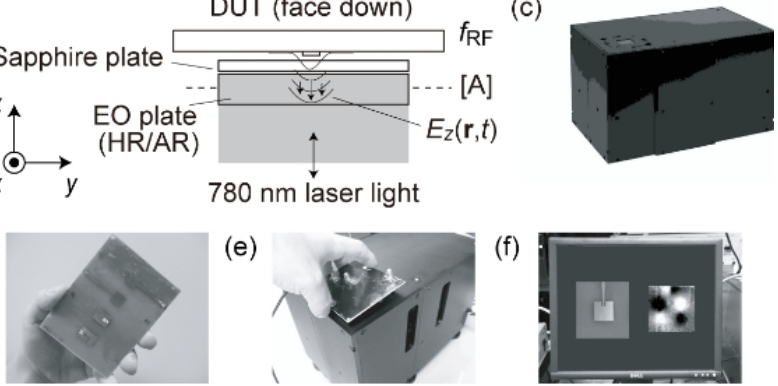

e)
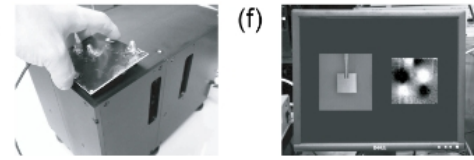

Fig. 1. (a) RF and optical configurations of LEI camera. AR: anti-reflection coating, CCD: charge coupled device, DM: dichroic mirror, HR: high-reflection coating, LED: light emitting diode, LO: local oscillator, PBS: polarization beam splitter, PC: personal computer. (b) Magnified cross-sectional view of device under test (DUT) and EO sensor plate is shown with a sensing laser beam and $z$ components of evanescent field $E_{z}$. (c) Latest prototype. (d) $\sim$ (f) Procedure for visual observation of a planar circuit.

\section{2-1 Configurations and Observation Procedure}

The LEI camera scheme relies on the ultrafast electrooptic (EO) effect in conjunction with ultraparallel complementary metal oxide semiconductor (CMOS) image sensor technology. A key for combining these two usually-conflicting "ultra" schemes is the spatially coherent process of frequency down-conversion in a LEI camera, which brings about a bandwidth of more than $100 \mathrm{GHz}$ at each pixel.

Fig. 1(a) schematically shows the RF and optical configurations of the LEI camera. A magnified drawing for the EO plate (ZnTe) and device under test (DUT) is in Fig. 1(b), in which a field $E_{z}(\mathbf{r}, t)$ at a frequency $f_{\mathrm{RF}}$ to be visualized is also illustrated. Here, $\mathbf{r}=(x, y)$ is a position in the EO plate plane indicated by $[\mathrm{A}]$ in the figure, and $t$ is time. DUT is assumed to be a pattern on a printed circuit board (PCB), whereas different kinds of DUTs are acceptable as described in III.

The area of the EO sensor plate is $25 \mathrm{~mm} \times 25 \mathrm{~mm}$.
The number of pixels for the CMOS image sensor in Fig. 1 (a) is $100 \times 100=10,000$, which gives an image resolution of $0.25 \mathrm{~mm} / \mathrm{pixel}$. The resolution is of course modified by the $z$-dependence of electrical field distributions as well as by the optical magnification factor described in 3-3.

Details of these configurations are in our previous publications [6] [8] and, therefore, only brief explanations are given here. An optical local oscillator (LO) signal is generated at a frequency $f_{\mathrm{LO}}$ with a $780 \mathrm{~nm}$ semiconductor laser and a Mach-Zehnder interferometer modulator. The laser light beam is then collimated and led through a polarization beam splitter (PBS) to the EO plate. After the interaction with evanescent electric field of DUT during a round trip of the laser light within the EO plate, the light goes back to PBS. The 2D phase modulation provided by the DUT electric field is transferred to $2 \mathrm{D}$ intensity modulation at PBS and resultant frequency down conversion provides a $2 \mathrm{D}$ optical signal at an intermediate frequency (IF) $f_{\mathrm{IF}}=f_{\mathrm{RF}}-f_{\mathrm{LO}}$. The $2 \mathrm{D} \mathrm{IF}$ signal is then detected by the CMOS image sensor with a sampling frequency $f_{\mathrm{IS}}$, which is approximately four times higher than $f_{\mathrm{IF}}$. Finally, the $2 \mathrm{D}$ signal is displayed as an image by a personal computer (PC).

Fig. 1(c) shows a photo of the latest prototype, which was designed for the mobile usage on a desktop. The bottom area of the box is as small as a sheet of A4 paper and its weight is approximately $15 \mathrm{~kg}$.

Fig. $1(\mathrm{~d}) \sim(\mathrm{f})$ show a typical procedure of visual observation for an RF wave over a planar circuit. It takes only a moment; take the PBC sample (Fig. 1(d)), place it on the camera (Fig. 1(e)), and see the image or movie on a PC display (Fig. 1(f)). Without this real-time feature, it would be impossible to perform such a high throughput for the ample images and movies reported previously [5] [12].

\section{2-2 Principle of Real-Time RF Wave Observations}

Two stages of 2D frequency down-conversion for the $\mathrm{RF}$ wave at $f_{\mathrm{RF}}$ are incorporated in the LEI camera system: first, electrooptically in the optics with respect to the optical LO signal at $f_{\text {LO }}$ and second, numerically in the digital signal processing (DSP) unit attached to the CMOS image sensor. The RF phase $\phi(\mathbf{r})$ at the plane [A] (Fig. 1(b)) is preserved during these processes and reproduced precisely in a final image in the PC display [C] (Fig. 1(a) and (f)) as follows. Let $E_{z}(\mathbf{r}, t)$ be expressed as

$$
\begin{aligned}
& E_{z}(\mathbf{r}, t)=A(\mathbf{r}) \cos \left(2 \pi f_{\mathrm{RF}} t-\mathbf{k} \cdot \mathbf{r}\right) \\
& \mathbf{k} \cdot \mathbf{r}=k_{x} x+k_{y} y=\phi(\mathbf{r})
\end{aligned} .
$$


Here, $A(\mathbf{r})$ and $\mathbf{k}=\left(k_{x}, k_{y}\right)$ are the amplitude and $2 \mathrm{D}$ wave vector of the RF wave, respectively. As described above, optical intensity modulation is generated by $E_{z}$ through irradiation of the optical LO signal to the EO plate (Fig. 1(b)) and the polarimetric optics in Fig. 1(a). The 2D distribution of the optical modulation at the surface plane [B] of the CMOS image sensor (Fig. 1(a)) is thus given by

$$
\begin{aligned}
& I\left(\mathbf{r}^{\prime}, t\right)=I_{0}+\Delta I\left(\mathbf{r}^{\prime}\right) \cos \left(2 \pi f_{\mathrm{IF}} t-\phi\left(\mathbf{r}^{\prime}\right)\right) \\
& \mathbf{r}^{\prime}=\left(x^{\prime}, y^{\prime}\right)
\end{aligned}
$$

$I, I_{0}$, and $\Delta I$ are the respective optical intensities for the total laser signal, the components of a continuous wave $(\mathrm{CW})$ as well as higher order modulations, and the component modulated at $f_{\mathrm{IF}} . \mathbf{r}^{\prime}=\left(x^{\prime}, y^{\prime}\right)$ denotes a position on the plane [B]. The optical signal given by (2) is the result of the first spatially coherent process of 2D frequency down-conversion. It is then detected and numerically processed in the DSP unit. The resultant image signal shown on the plane $[\mathrm{C}]$ is given by

$$
\begin{aligned}
& E_{z}^{\text {(image) }}\left(\mathbf{r}^{\prime \prime}, t\right) \propto A\left(\mathbf{r}^{\prime \prime}\right) \cos \left(2 \pi \delta f t-\phi\left(\mathbf{r}^{\prime \prime}\right)\right) \\
& \mathbf{r}^{\prime \prime}=(i, j)
\end{aligned}
$$

Here, $\mathbf{r}^{\prime \prime}=(i, j)$ is the pixel address and $\delta f$ is the difference frequency between $f_{\mathrm{IF}}$ and $f_{\mathrm{IS}} / 4$. Thus, $\delta f$ originates from the second step of the $2 \mathrm{D}$ frequency down-conversion and gives a frequency of phase evolution in a LEI movie. As indicated by (1) (3), the $2 \mathrm{D}$ phase relationship of the RF wave in the EO plate, i.e. wave properties, is reproduced precisely in the final LEI view, provided that the physical length on the EO plate is appropriately converted to a distance on the PC display. The real-time optical monitor of a sample surface is beneficial for this purpose. This is provided by a built-in charge-coupled device (CCD) camera, a 640-nm light emitting diode (LED) illuminator, and the dichroic feature of high-reflection coating on the EO plate and a mirror.

A visual representation as an LEI movie for (3) is called a "phasor" movie and displays the phasor characteristics at each pixel. It is useful for visualizing $\mathrm{RF}$ wave propagation. LEI movies for $A\left(\mathbf{r}^{\prime \prime}\right)$ and $\phi\left(\mathbf{r}^{\prime \prime}\right)$ are called "magnitude" and "phase" movies, respectively. Besides the above formulation for $E_{z}$ observation, $E_{\mathrm{x}}$ and/or $E_{\mathrm{y}}$ observations are possible with different crystallographic orientations of EO plates employed.

\section{Visual Observation Results}

Here, six examples of LEI camera experiments are presented, varying in their visual accesses to RF waves.

\section{3-1 Ku-Band Waves on Arrayed Planar Antennas}

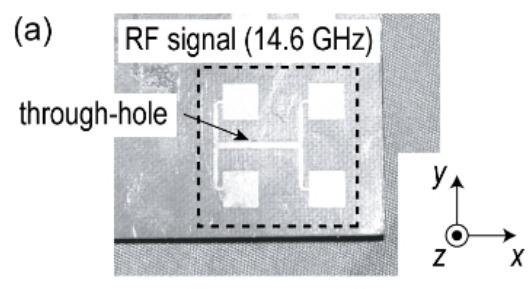

(b)

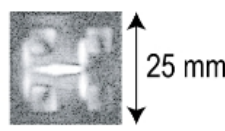
$\left|E_{Z}\right|(\mathrm{dB})$ $0 \quad 40$

(c) 1
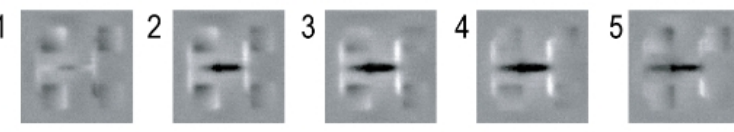

6

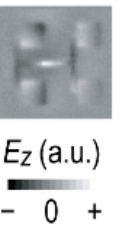

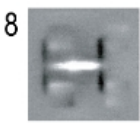
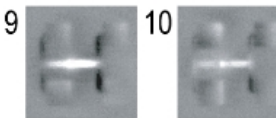

$E_{z}$ (a.u.)
$-0+$

Fig. 2. Visualization of RF waves on arrayed planar antennas. (a) Optical image of the antenna with an arrow indicating the position of through-hole (feed point) for the RF signal injection. LEI observation result for the area indicated by a dotted square in (a) is indicated by a magnitude image (b) and a stroboscopic series of phasor images with a phase interval of $\pi / 5$ (c).

Fig. 2 shows an $E_{z}$ observation result for arrayed planar antennas emitting a 14.6-GHz signal. Fig. 2(a) indicates the pattern of the antenna patches formed on a ceramic-polytetrafluoroethylene composite laminate board (Rogers, RT/duroid-6010LM), whose relative permittivity and thickness are 10.2 and $0.64 \mathrm{~mm}$, respectively. The Ku-band signal was fed via a through-hole and a subminiature type A (SMA) receptacle mounted on the back of the board. The position of the through-hole is indicated by an arrow in Fig. 2(a).

Each patch was designed to be identical and set symmetrically so that it works equally. However, the visualized result in Fig. 2(b) and (c) shows that standing waves on the four patches differ apparently. It is probably due to fabrication errors. Note that this kind of near-field visualization for arrayed planar antennas is useful because DUTs contain only one port and two-port network analysis, the most standardized method, is not applicable.

\section{3-2 Waves on a Gb/s-Class Digital Circuit [7]}

Fig. 3 shows an $E_{x}$ observation result for an intersectional part of a high-speed digital circuit: two-step prescaler. This sample provides an example of digital circuit that malfunctions in a few clock frequency ranges. It includes JK flip-flop integrated circuit chips of emitter-coupled logic (ECL). The circuit pattern was formed on a flame retardant type-4 (FR-4) glass epoxy circuit 
(a)
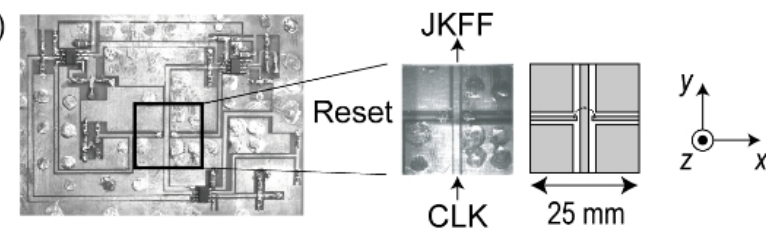

(b)

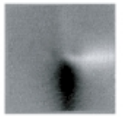

(c)
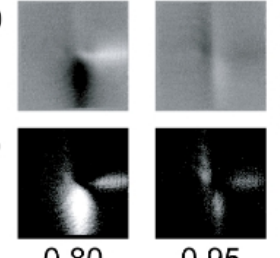

0.80

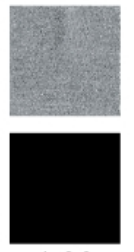

1.90 (H.I.)
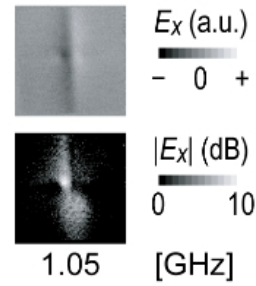

$[\mathrm{GHz}]$

Fig. 3. Visualization of waves on a Gb/s-class digital circuit. (a) Optical image of the whole circuit with a CCD image and a scheme for the circuit pattern of interest: the intersection of clock (CLK) and reset lines. Corresponding phasor and magnitude images are in (b) and (c), respectively. Higher order (second) harmonic images (H. I.) are shown for the $0.95-\mathrm{GHz}$ clock. JKFF: JK flip-flop.

board (Sunhayato), whose relative permittivity and thickness are 4.7 at $1 \mathrm{MHz}$ and $1.6 \mathrm{~mm}$, respectively.

The reset line bridges over the clock line on the PCB backside, as illustrated by the inset next to the CCD image in Fig. 3(a). Note in Fig. 3(b) and (c) that the signals stay on the reset line at clock frequencies 0.80 and 0.95 $\mathrm{GHz}$, which should not under an ideal operation condition. These images suggest presence of undesired couplings at the intersection, which seem to prevent further propagation of the clock signal. On the other hand, the coupling is scarce at $1.05 \mathrm{GHz}$. Indeed, a correspondence of frequency dependence was found between this clock signal behavior and the frequency characteristics of the whole circuit operation.

It has been thus demonstrated that an origin of malfunction for a high-speed digital circuit is visually found out. In addition, the visualization functionality of higher harmonics of digital signals is demonstrated as shown in Fig. 3(b) and (c); a 1.90-GHz wave appears in a phasor image in response to a launched $0.95-\mathrm{GHz}$ clock signal. This observation suggests possible distortion of digital signal waveforms since an ideal square wave contains no second-harmonic components.

\section{3-3 W-Band Waves [8]}

Fig. 4 shows results of $E_{z}$ observations for a W-band wave. It travels along the EO sensor plate, which acts as a semiconductor slab waveguide. A schematic for the experimental setup is in Fig. 4(a); the W-band wave is launched from an opening of a WR-10 flange into the

(a)

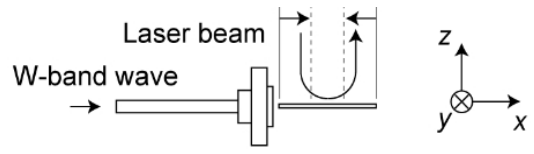

(b)

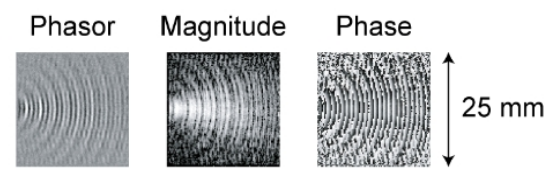

(c)

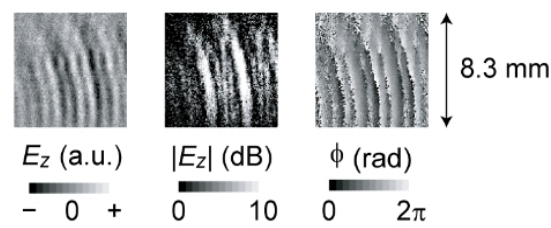

Fig. 4. W-band wave injected into a semiconductor slab waveguide: a ZnTe EO sensor plate. The experimental setup is shown schematically in (a). The dotted lines indicate the reduced waist of sensing laser light beam for the optical magnification of RF wave images. The traveling wave appears with optical magnification factors (b) 1 and (c) 3 .

EO sensor plate. For this experiment, the direction of laser beam propagation was set in the reverse direction of that shown in Fig. 1 for the sake of experimental convenience. As indicated by dotted lines and arrows in Fig. 4(a), the sensing laser beam can be narrowed by replacement of the optics set of the LEI camera system, which leads to optically expanded images. Thus, Fig. 4(b) and (c) shows observed images with optical magnifications of 1 and 3 , respectively.

Indicated clearly in Fig. 4(b) is that the W-band wave travels showing its wave fronts of concentric circles. The measured wavelength of this guided wave is approximately $1 \mathrm{~mm}$, which coincides with an estimated value from the relative permittivity of the EO material. Thus, visual accesses to the traveling W-band waves in a slab waveguide have been demonstrated.

On the other hand, there exist waves of a longer wavelength, which is approximately $3 \mathrm{~mm}$. Furthermore, a periodic variation is indicated in the magnitude image, which should be smoother if only the W-band wave is in a single slab waveguide mode. It is thus suggested that some mixture occurs between the slab waveguide mode and the aerially traveling mode. It is indicated more clearly in Fig. 4(c). It is also noticeable in its phasor image that a wave reflected at the end facet of the EO sensor plate appears.

Fig. 5 indicates another $E_{z}$ observation result for a W-band wave, where visual accesses to an aerially traveling wave are focused. Indeed, a W-band wave reflecting at the surface of $\mathrm{Cu}$ foil placed beneath the EO plate has been successfully visualized. 


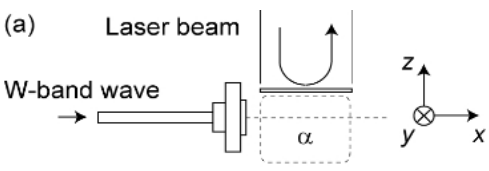

(b)

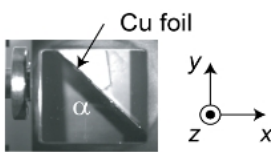

(c)

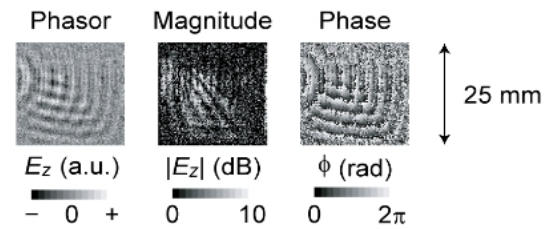

Fig. 5. W-band wave reflecting at a surface of $\mathrm{Cu}$ foil. The experimental setup is shown schematically in (a) and by a CCD image in (b). The reflection event images are in (c).

The experimental setup is indicated in Fig. 5 schematically (a) and photographically (b), where the wave emitted from an opening of a WR-10 flange propagates in the free space a toward the $\mathrm{Cu}$ foil glued on a triangle Styrofoam block. Fig. 5(c) shows a 2D interference pattern containing an aerially traveling wave and a reflected wave. However, pointed discussion is needed in order to distinguish each wave component from the other as well as from residual wave components because of their highly sophisticated features. In addition, a condition for optimized visual accesses to a specific wave component should be clarified [9].

\section{3-4 Backward-Traveling Wave along CRLH-TL [10]}

Fig. 6 shows an $E_{z}$ observation result for waves in a composite right-/left-handed transmission line (CRLHTL); a representative metamaterial structure. Its pattern formed on an FR-4 board is indicated in Fig. 6(a), which consists of nine periods of unit cells (Fig. 6(b)). An RF signal is injected from the top left to the bottom right as indicated by the arrow in the CCD image in Fig. 6(c). A text book set of images has been acquired, which had never been experimentally obtained; the wave pro- pagates oppositely as a backward wave at $1.8 \mathrm{GHz}$ (Fig. 6(d)) whereas it propagates forward at $4.0 \mathrm{GHz}$ (Fig. 6 (e)).

It should be noticed that a wavelength $\lambda$ can be measured directly on an image. Furthermore, a corresponding propagation constant $\beta=2 \pi / \lambda$ can be evaluated and such evaluations against frequencies leads to dispersion characterization. Fig. 7(a) and (b) show an example of the wavelength measurement for the wave on the CRLH-TL sample and plots of evaluated $\beta$ against the frequency $f_{\mathrm{RF}}$, respectively. The plot shows the dispersion characteristics of the CRLH-TL sample. Solid circles are for the forward waves whereas solid diamonds are for the backward waves. Here, it is apparent that the phase (a)

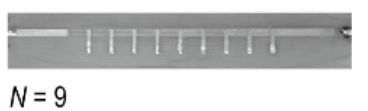

(c)

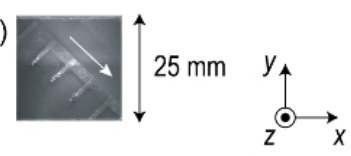

(b)

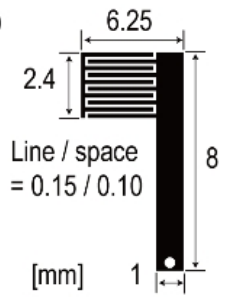

(d) 1
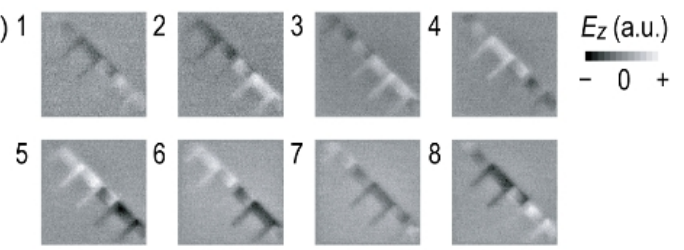

(e)
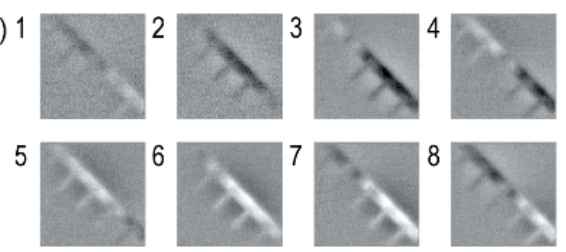

Fig. 6. Waves along a composite right-/left-handed transmission line (CRLH-TL). (a) Photo of the CRLHTL pattern including nine periods $(N=9)$ of unit cells indicated in (b). (c) CCD image taken in situ through the EO plate. Stroboscopic series of phasor images with a phase interval of $\pi / 4$ for frequencies of $1.8 \mathrm{GHz}(\mathrm{d})$ and $4.0 \mathrm{GHz}(\mathrm{e})$.

velocity $2 \pi f_{\mathrm{RF}} / \beta$ is negative for the backward propagation. In addition, phasor images at characteristic frequencies are shown so that their wave features are indicated visually [8]. Their detailed analyses would be a future work.

Solid curve in Fig. 7(b) indicate a reference, which is unwrapped phase data measured by a network analyzer [13]. Here, the frequency for $\beta=0$ is assumed to be 2.7 $\mathrm{GHz}$. Most of its characteristic features coincide fairly well with those of the dispersion plot whereas some discrepancies exist. It would be interesting to clarify the origin of the discrepancies and to discuss about the pros and cons between those methods, which will be a future work.

\section{3-5 Waves in MMIC Module Case [11]}

Fig. 8 shows preparation and $S$-parameter characterization result of a module containing a monolithic microwave integrated circuit (MMIC) chip. The chip is a commercially available traveling wave amplifier (AMMC5026, Avago Technologies). Its bandwidth and gain are 2 $35 \mathrm{GHz}$ and $10.5 \mathrm{~dB}$, respectively. Fig. 8(a) shows the bonding configuration of the module. Photos of the chip input, output, a DC decoupling capacitor located between two microstrip lines, and assembled module are 
(a)

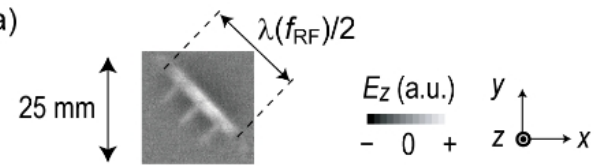

(b)

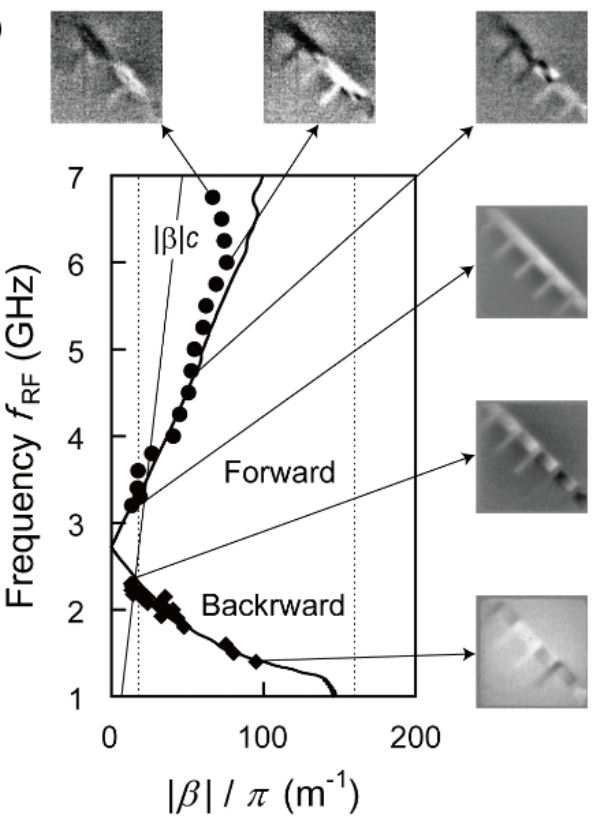

Fig. 7. Result of dispersion evaluation for the CRLH-TL sample in Fig. 6. (a) A schematic for the wavelength measurement. (b) Dispersion curves derived by the wavelength measurement (solid diamonds and solid circles) and by the network analyzer measurement (solid curve). Phasor images at characteristic frequencies are also indicated. $c$ is the velocity of light.

shown in Fig. 8(b), (c), (d), and (e), respectively. The result of its preliminary $S$-parameter measurement is in Fig. 8(f), in which magnitudes of $S_{21}$ are plotted with the drain voltage $V_{\mathrm{dd}}$ as a parameter.

Note that the module was designed for this particular demonstration for the visual observation and is not optimized for the maximized module performance. Indeed, the $\left|S_{21}\right|$ value is less than the specified gain value of the MMIC chip, which implies a possibility that the assembly of the module case leads to the degradation. It is also notable that concave portions exist around $28 \mathrm{GHz}$ for all the $\left|S_{21}\right|$ curves, despite the $V_{\text {dd }}$ value. This feature suggests a possible cavity resonance effect at this frequency.

Fig. 9 shows the setup and an $E_{z}$ observation result for the module. The propagation direction of the laser beam was again set inversely and the EO plate was inserted into the module case to the step frame at the bottom, as indicated in Fig. 9(a). A CCD image is shown in Fig. 9(b) and corresponding $E_{z}$ images are shown in Fig. $9(\mathrm{c}) \sim(\mathrm{g})$.
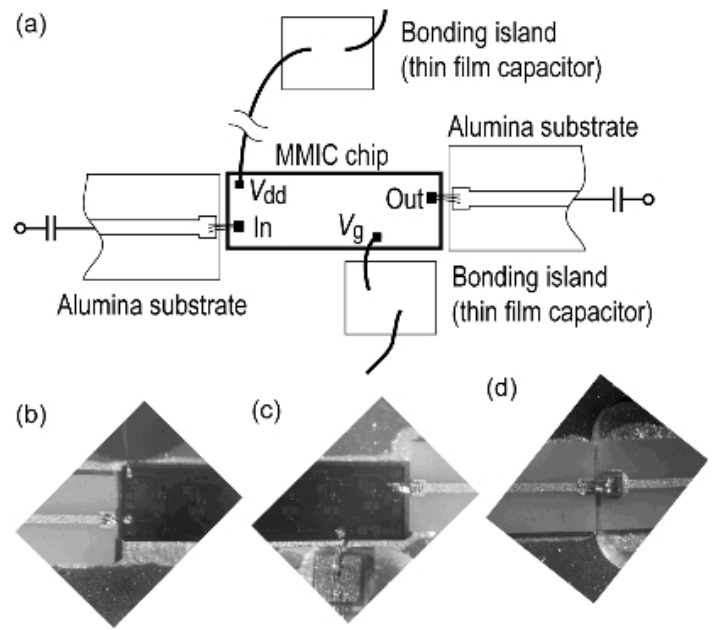

(e)

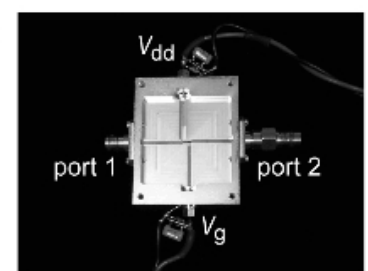

(f)

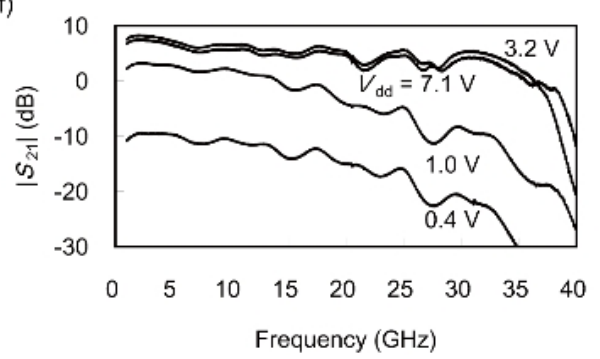

Fig. 8. Millimeter-wave MMIC module preparation. Bonding configuration (a) is shown with photos of chip input (b), chip output (c), and decoupling capacitor (d). Photo of the assembled module (e) and $S_{21}$ measured for the module with a variety of $V_{\mathrm{dd}}$ (f) are shown.

Three optical magnification factors were examined with a 15-GHz signal as shown in Fig. 9(c), (d) and (e). Taking the spatial resolution and size for the field of vision into account, magnification factor $\times 3$ was chosen as typical.

It is notable in Fig. 9(d) that the input and output signals are well-visualized whereas fields appear at the bonding wires and pads for the $V_{\mathrm{dd}}$ and $V_{\mathrm{g}}$ supply, which should be isolated from the RF waves. A peculiar feature also appears in the image at $28 \mathrm{GHz}$ (Fig. 9 (f) and (g)): a laterally spread wave travels back and forth in the case of the module. In line with the expectations considering the $S$-parameter features, it should be concluded that visualization of a cavity resonance phenomenon was demonstrated.

In cases of future real applications of this scheme, 
(a)

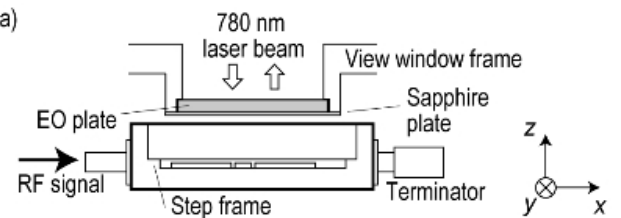

(b)
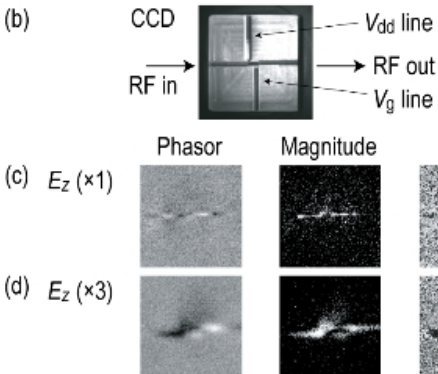

(e) $E_{z}(\times 10)$

(f) $28 \mathrm{GHz}$
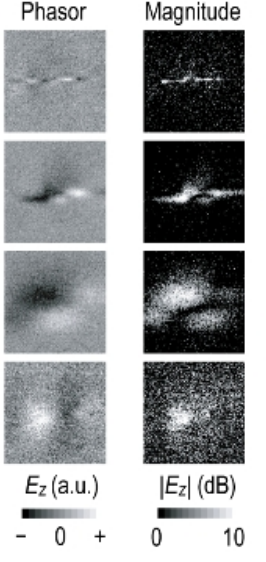

(g) Phasor $28 \mathrm{GHz}$
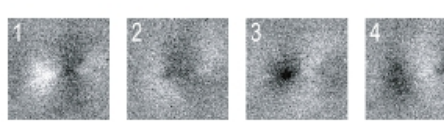

Fig. 9. Setup and result of visual observations for waves in MMIC module case. The phase interval for $(\mathrm{g})$ is $2 \pi / 5$.

however, it is indispensable to innovate in the LEI camera system so that the EO sensor plate and its holder can be adaptively inserted into a module case.

\section{Conclusion}

A wide variety of LEI approaches have been shown for visual accesses to $\mathrm{GHz}$ traveling electric waves. Further extensions of the scheme along this trend would lead to creation of a useful tool to promptly analyze and diagnose RF wave properties, which would be applicable to the GHz EMC issues. Room also remains for improving the LEI technique; observation area (size of the EO sensor plate), sensitivity, adaptability of the sensor head, analytical methods of images/movies and others. Their intensive improvements may provide new area of RF wave visualizations.

The authors thank Mr. Takahiro Hashiba, Dr. Kiyotaka Sasagawa, Dr. Atsushi Kanno, and Mr. Shigeki Morisawa for their assistance on the LEI observation work, and express their appreciation to Dr. Yoshihiro Imajo, Mr. Shinichi Shirasu, Prof. Toshi fumi Morimoto, and Dr. Yusuke Kusama for their support and discussion. The authors also thank Prof. Joji Hamasaki for his encouragement.

\section{References}

[1] K. Yang, et al., "Electrooptic mapping and finite-element modeling of the near-filed pattern of a microstrip patch antenna," IEEE Trans. Microwave Theory and Tech., vol. 48, pp. 288-293, 2000.

[2] E. Yamazaki, S. Wakana, H. Park, M. Kishi, and M. Tsuchiya, "High-frequency magneto-optic probe based on BiRIG rotation magnetization," IEICE Trans. Electron., vol. E86-C, pp. 1338-1344, 2003.

[3] K. Sasagawa, M. Tsuchiya, "Real-time monitoring system of RF near-field distribution images on the basis of 64-channel parallel electro-optic data acquisition," IEICE Electronics Express, vol. 2, pp. 600-606, 2005.

[4] K. Sasagawa, A. Kanno, T. Kawanishi, and M. Tsuchiya, "Live electro-optic imaging system based on ultra-parallel photonic heterodyne for microwave near-fields," IEEE Transaction of Microwave Theory and Techniques, vol. 55, pp. 2782-2791, 2007.

[5] M. Tsuchiya, R. Ikeno, "65,536-parallel measurements of phase resolved electric fields in $\mathrm{C}-\mathrm{K}$ bands and their applications to real-time visualization of $\mathrm{RF}$ waves," Proceedings of the 41st European Microwave Conference (EuMC2011), Manchester, EuMC234, pp. 41-44, Oct. 2011.

[6] K. Sasagawa, A. Kanno, and M. Tsuchiya, "Realtime digital signal processing for live electro-optic imaging," Optics Express, vol. 17, issue 18, pp. 1564115651, Aug. 2009.

[7] M. Tsuchiya, T. Shiozawa, "RF wave visions for circuit analyses and diagnoses by live electrooptic imaging camera," International Journal of Microwave and Wireless Technologies, vol. 2, pp. 341-347, 2010.

[8] M. Tsuchiya, K. Sasagawa, A. Kanno, and T. Shiozawa, "Live electrooptic imaging of W-band waves," IEEE Transaction of Microwave Theory and Techniques, vol. 58, issue 11, part 2, pp. 3011-3021, Nov. 2010.

[9] T. Shiozawa, M. Tsuchiya, "Real-time visualization of aerially propagating W-band radio waves," Proceedings of the 41st European Microwave Conference (EuMC2011), Manchester, EuMC03-1, pp. 10-13, Oct. 2011.

[10] M. Tsuchiya, T. Hashiba, and T. Shiozawa, "Visual observations of characteristic behaviors of RF waves in CRLH-TLs and their applications to dispersion characterization," IEEE Transaction of Microwave Theory and Techniques, vol. 58, issue 12, part 
2, pp. 4094-4101, Dec. 2010.

[11] M. Tsuchiya, T. Shiozawa, "Visual observation of internal signal transmissions in a millimeter-wave amplifier module," Proceedings of the 40th European Microwave Conference (EuMC2010), Paris, EuMC24-1, pp. 393-396, Sep. 2010.

Masahiro Tsuchiya

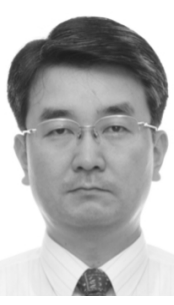

was born in Shizuoka, Japan, on Sep. 28, 1960. He received his Ph.D. degree in electronic engineering from the University of Tokyo, Japan, in 1988. In 2003, he joined the National Institute of Information and Communications Technology (NICT), Tokyo, Japan, and is presently an NICT executive researcher. His research interests include electronics and optoelectronics associated with info-communication systems and societies. Dr. Tsuchiya is a member of the Institute of Electronics, Information, and Communication Engineers, Japan, and the Japan Society of Applied Physics.
[12] Homepage of the live electrooptic imaging technique: http://lei-camera.nict.go.jp/

[13] C. Caloz, T. Itoh, Electromagnetic Metamaterials: Transmission Line Theory and Microwave Applications, Wiley, pp. 127-132, 2006.

Takahiro Shiozawa

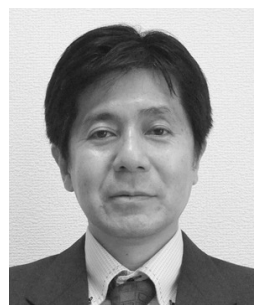

was born in Tokyo, Japan, on Dec. 14, 1957. He received his M.S. and Ph.D. degrees in electrical engineering from the University of Tokyo, Japan, in 1982 and 2005, respectively. In 1982, he joined the Yokogawa Electric Corporation, Tokyo, Japan. In 1990, he joined the Opto-Electronics Research Laboratories, NEC Corporation, Kanagawa, Japan. He is now with the Kagawa National College of Technology, Kagawa, Japan. His research interests include optical electronics and optical communication systems. Dr. Shiozawa is a member of the Institute of Electronics, Information, and Communication Engineers, Japan, and the Japan Society of Applied Physics. 